\title{
The Design and Implementation of a Single-Actuator Soft Robot Arm for Lower Back Pain Reduction
}

\author{
Alaa Al-Ibadi* \\ Computer Engineering Department, Basrah University, Basrah, Iraq
}

\author{
Correspondence \\ *Alaa Al-Ibadi \\ 61004 Basrah University, Basrah, Iraq \\ Email: alaa.abdulhassan@uobasrah.edu.iq
}

\begin{abstract}
This paper presents a simple and fast design and implementation for a soft robot arm. The proposed continuum arm has been built by a single self-bending contraction actuator (SBCA) with two-fingers soft gripper. Because of the valuable advantages of the pneumatic artificial muscle (PAM), this continuum arm provides a high degree of safety to individuals. The proposed soft robot arm has a bending behaviour of more $180^{\circ}$ at $3.5 \mathrm{~kg}$, while, its weight is $0.7 \mathrm{~kg}$. Moreover, it is designed to assist the people by reducing the number of backbends and that leads to a decrease in the possibility of lower back pain.

KEYWORDS: Pneumatic artificial muscle (PAM), Bending actuator, Lower back pain, Continuum arm, Human-robot interaction.
\end{abstract}

\section{INTRODUCTION}

The major cause of disabilities for a long period in life is the lower back pain (LBP) [1]. While more than $90 \%$ of LBP symptoms are unknown, clinical researchers are still developing structural anatomical and the mathematical biomechanical model [2]. On the other hand, the majority of work types require body bend to pick up tools and objects. This process causes medical back problems.

The high probability of injury risks is the major significant aspect of human-robot collaboration. The soft robotics represent substantial alternatives for rigid robots due to its softness and low weight. The soft pneumatic actuators that inspired by a human's muscle, such as the pneumatic artificial muscle (PAM), are widely utilised to build such forms of robotic systems. Numerous advantages are known for the PAM, such as the high force to weight ratio, multiple degrees of freedom (DoF), variable stiffness, low cost and it is safe for human-robot collaboration [3][4][5][6]. Thus, soft robots are safe for individuals and can work together with a human in the same workspace.

The bending pneumatic air muscles have been developed by numerous researchers. Among them is that presented by [7] using two chambers instead of one inner tube to create a bending actuator. The authors in [8] proposed a PneuNet actuator by utilising various thicknesses for the inner tube. The implementation of the PneuNet is easy, however, it lacks in the ratio of the elasticity. To overcome this restriction, a polymer fibre is used in the PneuFlex actuator to support the rubber substrate, as proposed by [9]. In comparison with the silicone, the polyethylene terephthalate
(PET) material is three to four times less elastic. [10] utilised the strain limiting layer on one side of the extension actuator to prevent elongation from this side and to make the other side free to extend. This method is used by [11] but adapted by using a high tension thread to partially fix the extensor PAM length. [12] utilised the impact of the braided angle by using two different braided angles of the braided mesh for the contractor muscle actuator to develop the bending performance. [13] used two integrated jamming actuators in parallel to create a bending performance. The jamming technique is used by [14] to control the bending stiffness.

[15] used tendons to establish the bending behaviour for the soft gripper. The PAM can be used in various engineering areas including humanoid robots, wearable robots for medical applications, industrial and airspace applications, and mobile robots [16]. On the other hand, bending muscle can be implemented by 3D printing, this technology provides fast prototyping, flexible design, and an easy way to implement the actuators and sensors that have the complex structure [17]. Furthermore, 3D printing offers an efficient way to build an actuator by using different materials and layout at the same time [18]. A fused deposition modelling (FDM) technology has been used by [19] to develop a 3D printed pneumatic muscle. Inserting soft sensors during the fabrication of the pneumatic actuators by the 3D technology is called 4D printing methods and it provides a valuable technique to manufacture such types of actuators [20].

In this paper, a proposal of a soft robot arm with a proper soft gripper is presented by using the techniques of the SBCA. The experiment to identify the performance of the proposed 
robot arm is explained together with several uses to assist the workers or lower back pain people.

\section{MATERIALS AND METHODS}

In this paper, the self-bending contraction actuator (SBCA) by Al-Ibadi, et al. [21] is used to design a single actuator continuum robot arm. The specifications for this actuator are listed in Table 1. The small size of the SBCA is used to design a two-finger soft gripper and it is mounted to the end of the soft robot arm.

\section{A. Fabrication of the continuum arm}

The simple type of the contraction pneumatic soft muscle is built by using inner rubber tube surrounded by braided mesh, then closed by two solid ends with a small air inlet [22] [23]. On the other hand, the SBCA is a modified version of the contraction actuator with an inserting flexible reinforcement road to prevent the contraction from the rod side and establishes the bending behaviour [24]. Fig. 1 shows the construction procedure of the continuum arm.

TABLE. 1.

The material specification of the robot continuum arm

\begin{tabular}{c|c}
$\mathrm{L}_{0}(\mathrm{~m})$ & 0.6 \\
\hline Rubber thickness $(\mathrm{m})$ & 0.0011 \\
\hline Braided thickness $(\mathrm{m})$ & 0.0005 \\
\hline Rubber diameter $(\mathrm{m})$ & 0.00265 \\
\hline Rubber stiffness(N/m) & 545 \\
\hline Rod length $(\mathrm{m})$ & 0.6 \\
\hline Rod thickness $(\mathrm{m})$ & 0.003 \\
\hline Rod width $(\mathrm{m})$ & 0.025
\end{tabular}

$L_{0}$ is the length of the SBCA at a relaxed condition (no pressure).

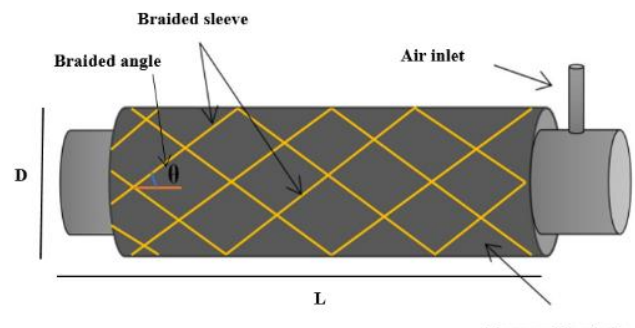

(a)

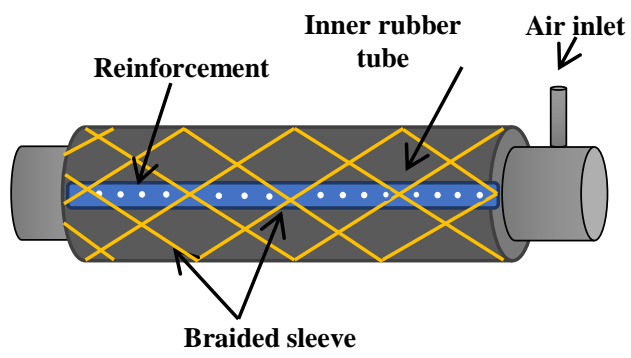

(b)

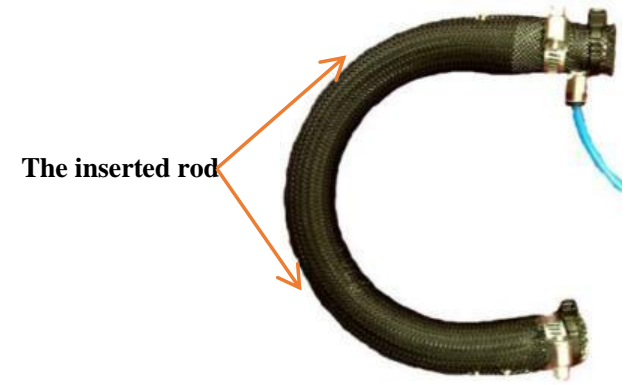

(c)

Fig. 1. The structure procedure of the continuum arm [21].

The end effector is made from two small size bending actuators. In order to maximise the grasping volume, the soft fingers are pulled by an elastomer ribbon as illustrated in Fig. 2.

The soft gripper offers highly efficient grasping performance due to its soft texture, compliance, high grasping force, low weight, and low cost. The end effector has been designed to be mounted at the end of the soft robot arm to grasp different types of objects and tools may the workers need to reduce the number of their back bending.

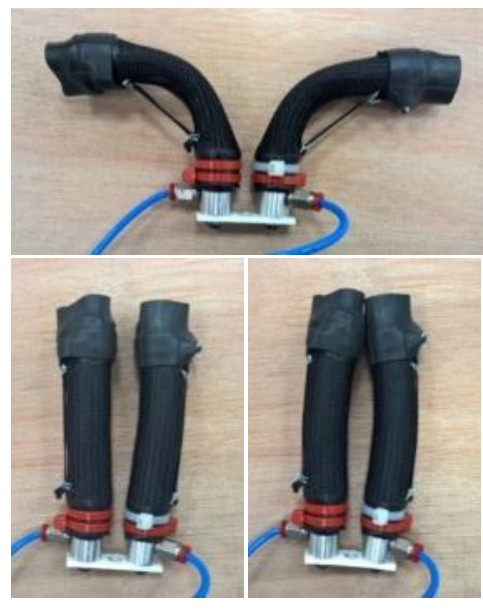

(a)

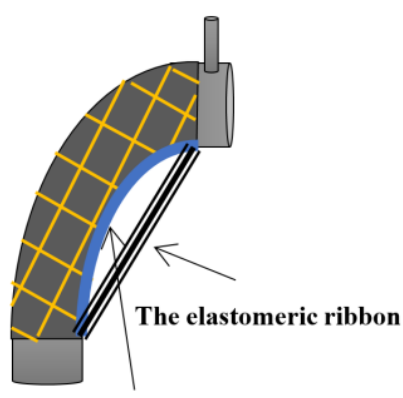

The reinforcement rod

(b)

Fig. 2. The two fingers soft gripper based on SBCA. (a) The soft gripper at different air pressures. (b) The schematic design for the soft finger. 
Fig. 3. Shows the continuum arm and the soft gripper at several actuating conditions. To verify the softness, compliance, and safety for human-robot interaction.

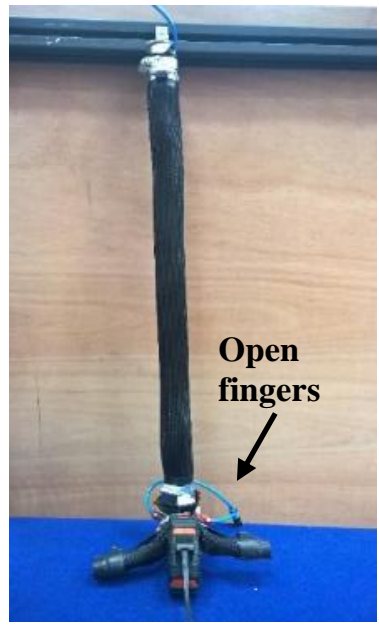

(a)

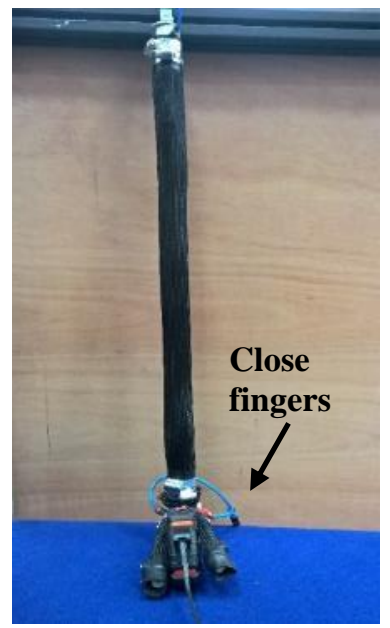

(b)

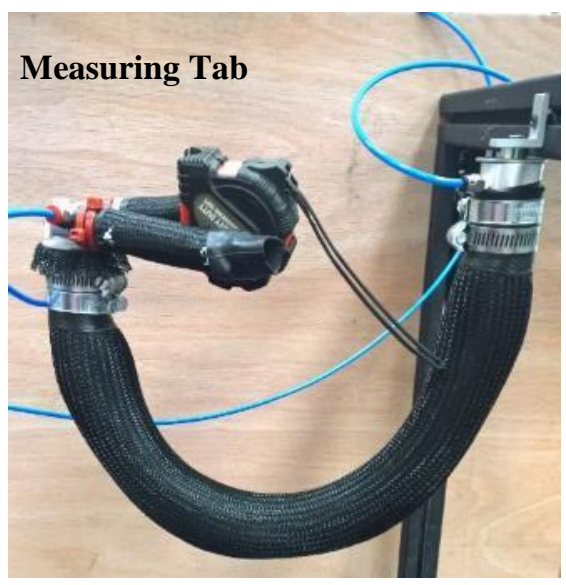

(c)
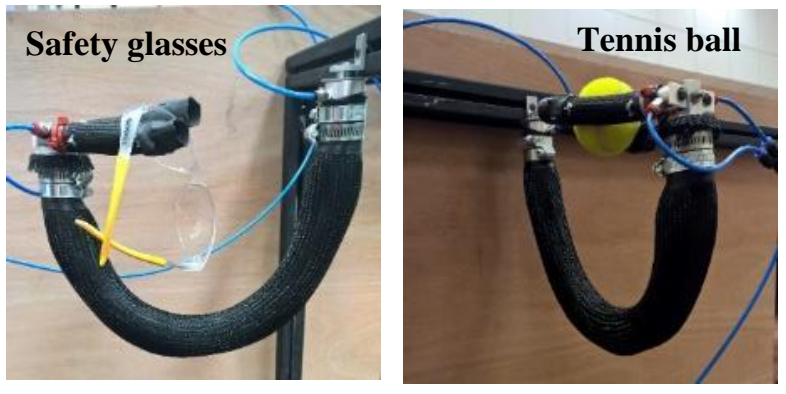

(d)

Fig. 3. The entire continuum arm and several actuations and grasping examples.

\section{EXPERIMENTS AND RESULTS}

An experiment has been done to evaluate the maximum bending force for the proposed arm. The continuum arm is fixed from the inlet end to the frame vertically, then air pressure is applied via three stages solenoid valve gradually. At each step, the soft arm bends to a specific bending angle (see Table. 2 For a $30 \mathrm{~cm}$ SBCA). To reduce the bending angle to its initial value (zero degrees), a load is attached to the free end. At each time the pressure is increasing, the load is rising to maintain zero bending angles. The maximum tested air pressure for safety work is $500 \mathrm{kPa}$. Fig. 4 shows an example of this procedure.

The proposed soft arm is tested at a maximum bending force of $9.2 \mathrm{~kg}$ and $3.5 \mathrm{~kg}$ at $180^{\circ}$. The weight of the proposed arm together with the two-fingers soft gripper is $0.7 \mathrm{~kg}$ and this gives about a " 13 " force to weight ratio.

TABLE. 2.

The maximum bending angle at different loads for the $30 \mathrm{~cm}$ SBCA

\begin{tabular}{c|c}
\hline Load $(\mathrm{kg})$ & Bending angle (degree) \\
\hline 0.0 & 213.1 \\
0.5 & 136.2 \\
1.0 & 73.0 \\
1.5 & 49.3 \\
2.0 & 34.1 \\
\hline
\end{tabular}

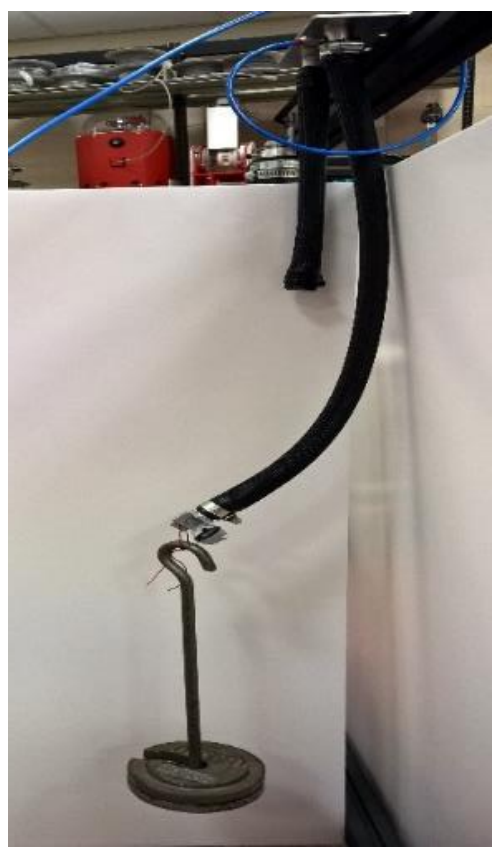

Fig. 4. A $30 \mathrm{~cm} \mathrm{SBCA} \mathrm{at} 300 \mathrm{kPa}$ and loaded by $1.0 \mathrm{~kg}$. 


\section{CONCLUSION}

Using the soft robot arm close to humans is safe due to the softness and the lightweight of its material.

The proposed robot arm provides significant assistance to humans to prevent or reduce the pain caused by bending the human back.

The pneumatic muscle actuator provides numerous advantages over the rigid types, Specially for the human-robot interaction.

This paper presented a single SBCA of $50 \mathrm{~cm}$ in length to design a robot arm that can be used close to humans to pick up objects and tools from the ground and lift them to the individuals to help them reduce the number of back bending. This leads to reducing back pain.

\section{ACKNOWLEDGMENT}

Many thanks to the computer engineering department of the university of Basrah for their support and providing the required lab facilities.

\section{CONFLICT OF INTEREST}

The authors have no conflict of relevant interest to this article.

\section{REFERENCES}

[1] D. Hoy et al., "The global burden of low back pain: Estimates from the Global Burden of Disease 2010 study," Ann. Rheum. Dis., 2014, doi: 10.1136/annrheumdis-2013-204428.

[2] R. A. Deyo, S. K. Mirza, J. A. Turner, and B. I. Martin, "Overtreating Chronic Back Pain: Time to Back Off?," J. Am. Board Fam. Med., vol. 22, no. 1, pp. 62-68, Jan. 2009, doi: 10.3122/jabfm.2009.01.080102.

[3] A. Al-Ibadi, S. Nefti-Meziani, and S. Davis, "The Design, Kinematics and Torque Analysis of the Self-Bending Soft Contraction Actuator," Actuators, vol. 9, no. 2, p. 33, Apr. 2020, doi: 10.3390/act9020033.

[4] K. Asaka and H. Okuzaki, Soft Actuators. Tokyo: Springer Japan, 2014.

[5] H. D. Yang, B. T. Greczek, and A. T. Asbeck, "Modeling and analysis of a high-displacement pneumatic artificial muscle with integrated sensing," Front. Robot. AI, 2019, doi: 10.3389/frobt.2018.00136.

[6] A. Al-Ibadi, S. Nefti-Meziani, and S. Davis, "Design, Kinematics and Controlling a Novel Soft Robot Arm with Parallel Motion," Robotics, vol. 7, no. 2, p. 19, May 2018, doi: 10.3390/robotics7020019.

[7] M. R. M. Razif, A. A. M. Faudzi, M. Bavandi, I. N. A. M. Nordin, E. Natarajan, and O. Yaakob, "Two chambers soft actuator realizing robotic gymnotiform swimmers fin," in 2014 IEEE International Conference on Robotics and Biomimetics (ROBIO 2014), 2014, pp. 15-20, doi: 10.1109/ROBIO.2014.7090300.

[8] F. Ilievski, A. D. Mazzeo, R. F. Shepherd, X. Chen, and G. M. Whitesides, "Soft robotics for chemists," Angew. Chemie - Int. Ed., 2011, doi: 10.1002/anie.201006464.
[9] R. Deimel and O. Brock, "A compliant hand based on a novel pneumatic actuator," in 2013 IEEE International Conference on Robotics and Automation, 2013, pp. 2047-2053, doi: 10.1109/ICRA.2013.6630851.

[10] G. Miron, B. Bédard, and J.-S. Plante, "Sleeved Bending Actuators for Soft Grippers: A Durable Solution for High Force-to-Weight Applications," Actuators, vol. 7, no. 3, p. 40, Jul. 2018, doi: 10.3390/act7030040.

[11] B. Tondu and P. Lopez, "Modeling and control of McKibben artificial muscle robot actuators," IEEE Control Syst., vol. 20, no. 2, pp. 15-38, Apr. 2000, doi: $10.1109 / 37.833638$.

[12] A. A. M. Faudzi, M. R. M. Razif, I. N. A. M. Nordin, K. Suzumori, S. Wakimoto, and D. Hirooka, "Development of bending soft actuator with different braided angles," in IEEE/ASME International Conference on Advanced Intelligent Mechatronics, AIM, 2012, doi: 10.1109/AIM.2012.6266037.

[13] A. Jiang, S. Adejokun, A. Faragasso, K. Althoefer, T. Nanayakkara, and P. Dasgupta, "The granular jamming integrated actuator," in 2014 International Conference on Advanced Robotics and Intelligent Systems (ARIS), 2014, pp. 12-17, doi: 10.1109/ARIS.2014.6871512.

[14] T. Wang, J. Zhang, Y. Li, J. Hong, and M. Y. Wang, "Electrostatic Layer Jamming Variable Stiffness for Soft Robotics," IEEE/ASME Trans. Mechatronics, vol. 24, no. 2, pp. 424-433, Apr. 2019, doi: 10.1109/TMECH.2019.2893480.

[15] M. Manti, T. Hassan, G. Passetti, N. D’Elia, C. Laschi, and M. Cianchetti, "A Bioinspired Soft Robotic Gripper for Adaptable and Effective Grasping," Soft Robot., vol. 2, no. 3, pp. 107-116, Sep. 2015, doi: 10.1089/soro.2015.0009.

[16] G. Andrikopoulos, G. Nikolakopoulos, and S. Manesis, "A Survey on applications of Pneumatic Artificial Muscles," in 2011 19th Mediterranean Conference on Control and Automation, MED 2011, 2011, doi: 10.1109/MED.2011.5982983.

[17] A. Zolfagharian, A. Z. Kouzani, S. Y. Khoo, A. A. A. Moghadam, I. Gibson, and A. Kaynak, "Evolution of 3D printed soft actuators," Sensors Actuators A Phys., vol. 250, pp. 258-272, Oct. 2016, doi: 10.1016/j.sna.2016.09.028.

[18] J. Z. Gul et al., "3D printing for soft robotics - a review," Sci. Technol. Adv. Mater., vol. 19, no. 1, pp. 243-262, Dec. 2018, doi: 10.1080/14686996.2018.1431862.

[19] H. K. Yap, H. Y. Ng, and C. H. Yeow, "High-Force Soft Printable Pneumatics for Soft Robotic Applications," Soft Robot., 2016, doi: 10.1089/soro.2016.0030.

[20] A. Zolfagharian, A. Kaynak, and A. Kouzani, "Closed-loop 4D-printed soft robots," Mater. Des., vol. 188, p. 108411, Mar. 2020, doi: 10.1016/j.matdes.2019.108411.

[21] A. Al-Ibadi, S. Nefti-Meziani, and S. Davis, "Active Soft End Effectors for Efficient Grasping and Safe Handling," IEEE Access, vol. 6, pp. 23591-23601, 2018, doi: 10.1109/ACCESS.2018.2829351.

[22] A. Al-Ibadi, S. Nefti-Meziani, and S. Davis, "Efficient 
Structure-Based Models for the McKibben Contraction Pneumatic Muscle Actuator: The Full Description of the Behaviour of the Contraction PMA," Actuators, vol. 6, no. 4, p. 32, Oct. 2017, doi: 10.3390/act6040032.

[23] S. Neppalli and B. A. Jones, "Design, construction, and analysis of a continuum robot," in 2007 IEEE/RSJ International Conference on Intelligent Robots and Systems, 2007, pp. 1503-1507, doi: 10.1109/IROS.2007.4399275.

[24] A. Al-Ibadi, S. Nefti-Meziani, S. Davis, and T. Theodoridis, "Novel Design and Position Control Strategy of a Soft Robot Arm," Robotics, vol. 7, no. 4, p. 72, Nov. 2018, doi: 10.3390/robotics7040072. 